\title{
Polyarteritis associated with Yersinia enterocolitica infection
}

\author{
Kevin Pile, Terry Kwong, Judith Fryer, Rodger Laurent
}

\begin{abstract}
A patient developed polyarteritis, predominantly affecting the muscles, 10 days after a Yersinia enterocolitica 0:3 infection. Immunoperoxidase staining showed Yersinia enterocolitica 0:3 antigen in the subendothelial layer of the blood vessels. This suggests that vasculitis should be considered as a rare manifestation of Yersinia enterocolitica infection.
\end{abstract}

Yersinia enterocolitica is a well recognised enteric pathogen in Europe and the USA. It is becoming increasingly important in Australia and is the third most common enteric pathogen after Campylobacter jejuni and Salmonella sp. ${ }^{1}$ Yersinia enterocolitica is associated with several postinfectious manifestations in $20 \%$ of infected patients. These occur predominantly in adults and include arthritis, erythema nodosum, pericarditis, carditis, and glomerulonephritis. ${ }^{2-4}$ There are only a few reports of an association between Yersinia enterocolitica and vasculitis. ${ }^{5-8}$

We report a case of polyarteritis associated with Yersinia enterocolitica 0:3 infection in which Yersinia enterocolitica $0: 3$ antigen was detected in the blood vessels.

\section{Case report}

A 43 year old man was admitted to hospital unable to walk because of severe generalised myalgia, most severe in the thigh and calf muscles, which had been present for three days. $\mathrm{He}$ also had frontal headache and photophobia.

Two weeks before admission he had had mild diarrhoea which lasted for 24 hours. This was associated with cramping abdominal pain and a mild myalgia which resolved after three days. He had been previously well and had no relevant medical history.

On physical examination, his temperature was $38^{\circ} \mathrm{C}$ and there was generalised muscle tenderness, particularly in the legs, with pain on stretching the muscles or isometric contraction. He was unable to fully extend the knees and elbows and dorsiflex the ankles because of muscle pain. There was no synovitis, lymphadenopathy, hepatosplenomegaly, cardiovascular, or respiratory abnormalities.

The haemoglobin was $13 \cdot 7 \mathrm{~g} / \mathrm{l}$, packed cell volume $0 \cdot 423$, leucocyte count $22 \cdot 1 \times 10^{9} / 1$ with 95\% neutrophils, $3 \%$ lymphocytes, and $2 \%$ monocytes, and platelet count $3.91 \times 10^{9} / 1$. The erythrocyte sedimentation rate (ESR, Westergren) was $58 \mathrm{~mm} /$ hour and $C$ reactive protein $399 \mathrm{mg} / \mathrm{l} . \alpha_{1}$ and $\alpha_{2}$ globulins were increased, albumin was $35 \mathrm{~g} / \mathrm{l}$, and creatine kinase $50 \mathrm{~g} / \mathrm{l}$. Serum IgG was $10.5 \mathrm{~g} / \mathrm{l}, \mathrm{IgA} 2.3 \mathrm{~g} / \mathrm{l}$, and $\operatorname{IgM}$ $1.42 \mathrm{~g} / 1$; cryoglobulins were not detected. Blood and stool cultures were negative and urine analysis and renal function were normal. Hepatitis B surface antigen was not detected and antibodies to Borrelia burgdorferi and Leptospira (8 serovars) were absent. Antibodies to streptolysin and DNase B were not increased. IgM antibodies to Yersinia enterocolitica O:3 showed an agglutination titre of 800 , to Yersinia enterocolitica 0:9 100, and Yersinia pseudotuberculosis type I less than 100. Titres of greater than 200 are diagnostically significant. A chest radiograph was normal and antibodies to neutrophil cytoplasmic antigen were not detected. His cerebrospinal fluid was normal.

It was initially considered that the patient was septicaemic but treatment with parenteral penicillin and gentamycin did not give any benefit. He was then treated with indomethacin $150 \mathrm{~g}$ per day, which gave a considerable improvement in symptoms and fever. The intravenous antibiotics were then discontinued and he was treated with doxycycline $200 \mathrm{mg}$ per day. When the indomethacin was discontinued, the fever and myalgia recurred.

An open biospy sample of the right gastrocnemius muscle showed a florid acute vasculitis affecting the small muscular arteries. The foci of fibrinoid necrosis in the vessel walls were associated with a dense cellular infiltrate composed of polymorphonuclear neutrophils, lymphocytes, histiocytes, and occasional multinucleate giant cells. Figure 1 shows that the appearances were consistent with polyarteritis nodosa.

Yersinia enterocolitica 0:3 antigens were subsequently detected in the walls of noninflamed small arteries by the immunoperoxidase technique using polyvalent antisera to Yersinia enterocolitica O:3 (Denka Seiken, Tokyo, Japan). ${ }^{9}$ Figure 2 shows that the most intense staining was in the subendothelial layer. There was no staining using antisera to Yersinia enterocolitica O:5 (Denka Seiken). The control sample was from a patient with polyarteritis nodosa affecting the calf muscles without evidence of Yersinia enterocolitica infection and no staining was seen. Sections from the patient and control which were processed substituting phosphate buffered saline for the primary antibody showed no staining.

Prednisone $25 \mathrm{mg}$ per day was begun and this gave a rapid improvement in symptoms. Two weeks later, the patient was asymptomatic with a haemoglobulin of $126 \mathrm{~g} / \mathrm{l}$, white cell count $11.7 \times 10^{9} / 1$, and an ESR of $22 \mathrm{~mm} /$ hour. The

Rheumatology,

Royal North

St Leonards, NSW 2065 Australia.

Accepted for publication 30 July 1991 
Figure 1 Florid acute vasculitis affecting $a$ small muscular artery, consistent with polyarteritis nodosa. Haematoxylin and eosin staining. $B a r=100 \mu \mathrm{m}$.

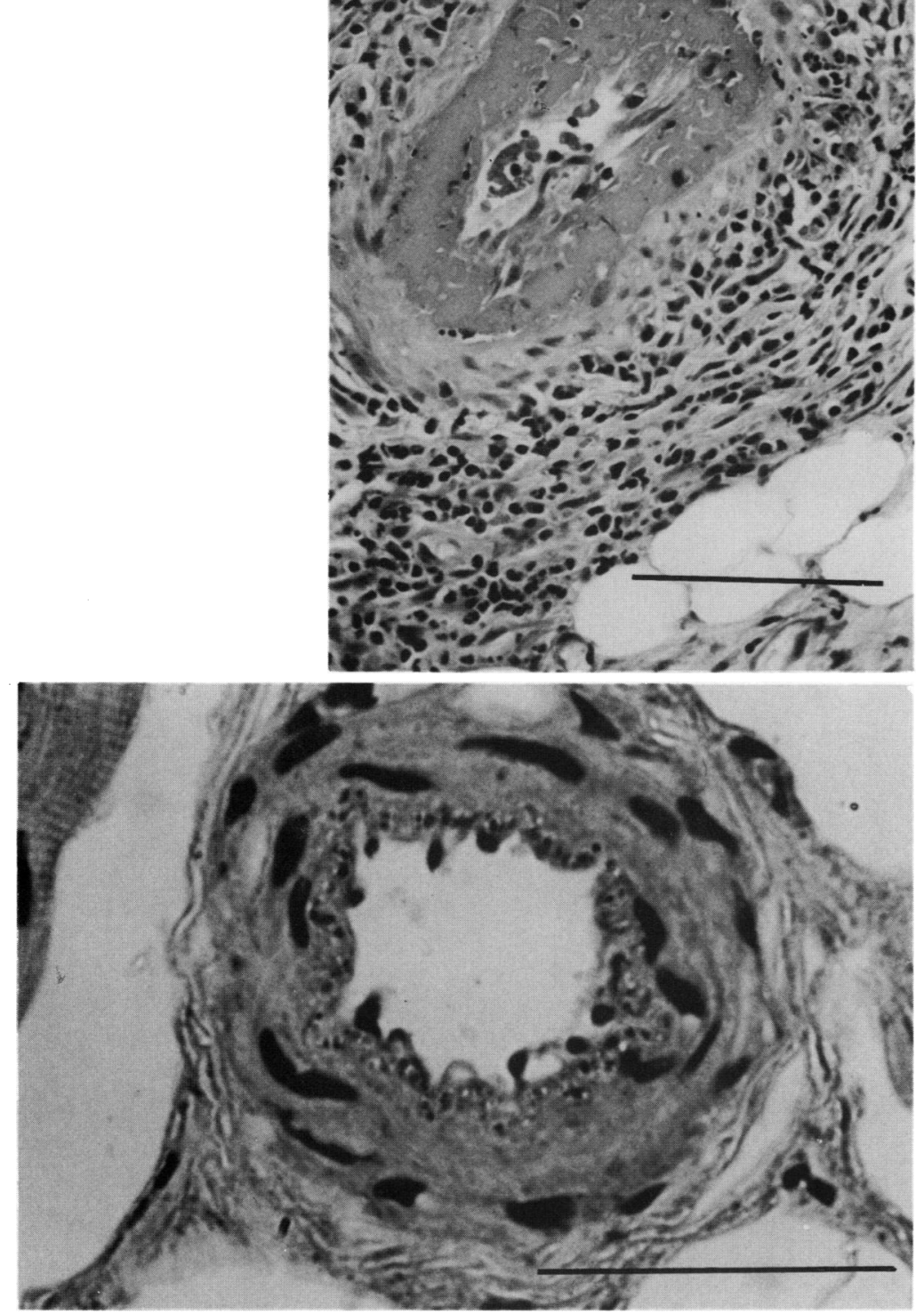

Figure 2 Immunoperoxidase staining of blood vessel showing Yersinia enterocolitica $O: 3$ antigen in the subendothelial layer. Bar $=50 \mu \mathrm{m}$.

patient has remained asymptomatic and eight months after the onset of symptoms, his maintenance treatment consists of prednisone $4 \mathrm{mg}$ per day. The doxycycline was stopped after two months. The haemoglobin is $152 \mathrm{~g} / \mathrm{l}$, white cell count $7 \cdot 4 \times 10^{9} / 1$, and ESR $2 \mathrm{~mm} /$ hour. The titre of IgM antibodies to Yersinia enterocolitica $0: 3$ is now less than 100 .

In summary, a 43 year old man developed polyarteritis predominantly affecting the muscles 10 days after an infection due to Yersinia enterocolitica O:3. Yersinia enterocolitica $0: 3$ antigens were detected in the blood vessels of the affected muscles.

\section{Discussion}

Yersinia enterocolitica is an organism that may persist within the body giving rise to chronic infection $^{10}$ or post-infection manifestations, particularly reactive arthritis. ${ }^{4}$ In patients with chronic infection, organisms have been detected in intestinal mucosa, lymph nodes, and the liver and, in particular, within the endothelial cells of the liver sinusoids. ${ }^{10}$ Yersinia enterocolitica antigen has been detected in synovial fluid cells from patients with reactive arthritis, which suggests that persistence of the antigen in the joint may be relevant to the arthritis. ${ }^{11}$ The polyarteritis in this patient was consistent with a post-infectious manifestation rather than due to direct infection and the presence of Yersinia enterocolitica antigen within the blood vessels suggests that this was an aetiological factor. Evidence for yersinia infection were the clinical features and increased antibody titre, although Yersinia enterocolitica was not cultured from the faeces $\mathbf{1 0}$ days after the onset of the illness.

The association between reactive arthritis and the genetic marker HLA-B27 suggests genetic susceptibility, ${ }^{12}$ but the numbers of patients affected are too small to determine whether this may apply to other manifestations of yersinia infections.

The staining was specific because it only occurred with antibody to the O:3 serotype and not with the 0:5 serotype and the control biopsy specimen of polyarteritis without preceding yersinia infection was also negative. Yersinia enterocolitica $0: 3$ antigen was found only in minimally or unaffected blood vessels and not in those with severe necrotic changes. This was probably because, in necrotic vessels, the antigen could not be detected as it was further destroyed by macrophages and neutrophils.

There is no direct evidence of an association between Yersinia enterocolitica and vasculitis. Serological evidence of infection with Yersinia enterocolitica associated with polyarteritis nodosa affecting the calf muscles has been reported. ${ }^{5}$ There have also been comments about a possible association between yersinia infection and Kawasaki disease based on the observation that some children with yersinia infection have features of Kawasaki disease. ${ }^{6} \mathrm{~A}$ child with Yersinia enterocolitica septicaemia had polyarteritis nodosa-like lesions in the blood vessels of the lung, liver, and kidney. ${ }^{7}$

Infection is recognised as a cause of polyarteritis nodosa, the most common organism being hepatitis $\mathrm{B}$, with $30 \%$ of people with polyarteritis nodosa having the hepatitis $B$ antigen in their serum. ${ }^{13}$ Other infectious agents that have been associated with vasculitis include cytomegalovirus, ${ }^{14}$ streptococcus, staphylococcus, and mycobacterium. ${ }^{813}$

In summary, we report a case of polyarteritis following Yersinia enterocolitica O:3 infection in which the Yersinia enterocolitica O:3 antigen was detected in the subendothelial layer of the blood vessels. Vasculitis could be considered as a rare manifestation of Yersinia enterocolitica infection occurring some time after the infectious event. 
3 Agner E, Larsen J H, Leth A. Yersinia enterocolitica carditis as a differential diagnosis-and the prognosis of this disease. Scand J Rheumatol 1978; 7: 26-8.

4 Laitinen $O$, Tuuhea J, Ahvonen P. Polyarthritis associated with Yersinia enterocolitica infection. Ann Rheum Dis 1972; 31: 34-9.

5 Brandrup F, Petersen E M, Hansen B F. Localised polyarteritis nodosa in the lower limb with new bone formation. Acta Dermatovener (Stockholm) 1980; 60: 182-4.

6 Larsen J H. Kawasaki disease-a Yersinosis? [letter]. 7 Infect Dis 1989; 160: 900.

7 Leonova L V. On morphology of Yersinia caused by Yersinia enterocolitica. Arkiv Pathologic Heskoi 1980; 42: 52-6.

8 Smail A, Ducroix J P, Tondriaux A, Sevestre H, Yzet T, Baillet J. Role de l'infection dans le declenchment des angeites necrosantes. Ann Med Interne 1988; 139: 324-30.

9 Gatter K C, Falini B, Mason D Y. The use of monoclonal antibiotics in histopathological diagnosis. In: Anthony P D, MacSween R N M, eds. Recent advances in histopathology, 12. Edinburgh: Churchill Livingstone, 1984.

10 Hoogkamp-Korstanje J A A, de Koning J, Heesemann J. Persistence of Yersinia enterocolitica in man. Infection 1988; 16: 81-5.

11 Granfors K, Jalkanen S, von Essen R, et al. Yersinia antigens in synovial fluid cells from patients with reactive arthritis. $N$ Engl $\mathcal{F}$ Med 1989; 320: 216-21.

12 Aho K, Ahvonen P, Lassus A, Sievers K, Tiilikainen A. HLA 27 in reactive arthritis. A study of yersinia arthritis and Reiter's disease. Arthritis Rheum 1974; 17: 521-6.

13 Cupps T R, Fauci A S. The vasculitides. Major problems in internal medicine. Vol XXI. Philadelphia: Saunders 1981: 27.

14 Doherty M, Bradfield J W B. Polyarteritis nodosa associated with acute cytomegalovirus infections. Ann Rheum Dis 1981; 40: 419-21. 